\section{Antikörper gegen GLP-1-Agonisten: Selten Kreuzreaktion mit GLP-1}

Es gibt nicht viele Kreuzreaktionen zwischen Antikörpern (AK) gegen einen der beiden GLP-1-Agonisten Liraglutid (Lira) und Exenatide (Exe) und dem körpereigenen Inkretinhormon GLP-1, aber es gibt sie. In der Studie LEAD 6 machten die Forscher anhand von Bluttests die Probe aufs Exempel. In der Studie folgte auf eine 26-wöchige Vergleichsphase zwischen Exe und Lira eine 52-wöchigen Phase, in der alle der ursprünglich 467 Patienten Lira erhielten. Es wurden mehrfach Blutproben entnommen und auf Kreuzreaktionen mit GLP-1 sowie auf den jeweiligen Wirkstoff neutralisierende Antikörper hin untersucht. Zu Studienbeginn wurde nur ein Patient positiv getestet.

Mit Lira wurden deutlich weniger Wirkstoff-AK produziert als mit Exe, was durch die größere Ähnlichkeit von Lira mit humanem GLP-1 erklärt wird. Nach 78 Wochen hatten vier der 154 in der Gruppe verbliebenen Patienten einen niedrigen AK-Titer gegen Lira, eine Probe hatte einen neutralisierenden Effekt auf den Wirkstoff in vitro, vier kreuzreagierten mit GLP-1. In der Gruppe, die zunächst Exe erhalten hatte, hatten 113 von 185 Patienten Wirkstoff-AK entwickelt, zwölf mit In-vitro-Neutralisierung, fünf mit GLP-1-Kreuzreaktion. Nach Umstellung auf Lira hatten jeweils drei Proben nach den übrigen 52 Wochen neutralisierende Lira-AK oder kreuzreagierten mit GLP-1. spa

Buse JB et al. Diabetologia 2010;53(Suppl 1) S341. P 857

\section{Fragiles Glukosegleichgewicht auch bei Gesunden}

Eine kurze Belastung mit hochkalorischer Ernährung, wenig Bewegung und Prednisolon setzt selbst bei Gesunden schon nach etwa zwei Wochen fatale Stoffwechselprozesse in Gang. Dies ergab eine Untersuchung mit zehn jungen Probanden ohne Risikofaktoren für Diabetes, allesamt Kaukasier. Bei innen wurde mit einer zehntägigen hochkalorischen Diät, physikalischer Inaktivität und $37,5 \mathrm{mg} / \mathrm{d}$ PrednisoIon eine Insulinresistenz ausgelöst. Dann maß man Glukoseparameter und die Inkretine GIP und GLP-1 nach einer flüssigen 520-kcal-Mahlzeit. Es stellte sich heraus, dass sich - ohne dass das Gewicht verändert war - sowohl die Nüchternglukose als auch die Insulinantwort erhöht hatten. Die verringerte Insulinsensitivität hatte keinen Einfluss auf das postprandiale GLP-1. Allerdings war GIP postprandial nun stark erhöht, ebenso Glukagon. Für GIP wird postuliert, dass es die Verbindung herstellt zwischen fettreicher Ernährung, Adipositas, Insulinresistenz und Typ-2-Diabetes und die Glukagonsekretion erhöht. Die Forscher schließen aus den Ergebnissen, dass das erhöhte GIP und die Hyperglukagonämie keine Ursachen, sondern Folgen der Insulinresistenz sind. Die erniedrigte GLP-1-Antwort dagegen scheint nicht von einer Insulinresistenz getriggert zu werden. spa Knop FK et al. Diabetologia 2010;53(Suppl 1) S260. P 647

\section{Strategien gegen Diabetes}

Das Entwickeln neuer Präventionsstrategien für Typ-1 und Typ-2-Diabetes sowie zur Vorhersage und Vorbeugung von Komplikationen, das sind Ziele des europäischen Projektes DIAMAP, das beim EASD in Stockholm vorgestellt worden ist. Das von der Europäischen Kommission geförderte Projekt wird koordiniert von der Alliance for European Diabetes Resarch (EURADIA). Darüber hinaus soll die Forschung zu Methoden, Diabetes zu verhüten und sogar zu heilen, unterstützt werden. Dieses Ziel richtet sich auf Wiederherstellung und Erhalt der Betazellfunktion. Auch der Transfer von Erkenntnissen der Grundlagenwissenschaft in klinischen Nutzen für Patienten soll gefördert werden. Dazu soll es eine europäische Plattform für klinische Diabetesfoschung geben. Auch die Ausarbeitung von Methoden zur Lebensstiländerung mit individualisierten Ernährungs- und Bewegungsempfehlungen gehört dazu. Und schließlich: Entwicklung und Validierung neuer Wege zur Prävention von und Behandlung bei mikro- und makrovaskulären Komplikationen.

Eine erste Bestandsaufnahme der Forschungsaktivitäten zu Diabetes in Europa hat ergeben, dass es ein erhebliches Defizit bei den Mitteln zur Diabetesforschung gibt. So werden jährlich 500 Millionen Euro dafür ausgegeben, die Kosten der Behandlung betragen jedoch 50 Millarden Euro jährlich. Zum anderen wird die Forschung in Europa kaum koordiniert. DIAMAP hat daher eine Online-

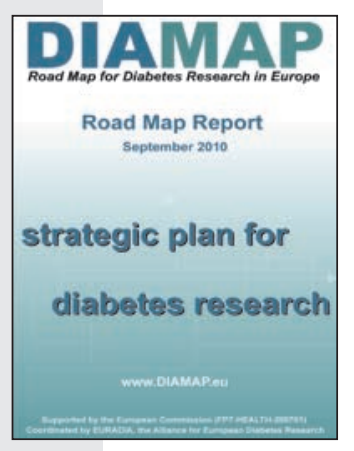
Datenbank entwickelt, mithilfe derer Forscher und Geldgeber potenzielle Möglichkeiten der Zusammenarbeit, der Leistungsfähigkeit und der Arbeitsgrundlagen erkennen können.

Mehr Informationen zum Projekt gibt es im Internet unter www.DIAMAP.eu und www.EURADIA.org

\section{Ein Jahr Insulin, zwei Kilo mehr auf der Waage}

Die Befürchtung vieler Typ-2-Diabetiker, nach dem Beginn einer Insulintherapie zuzunehmen, hat sich in einer Metaanalyse meist bestätigt. In der Untersuchung aus 51 randomisierten Parallelgruppenstudien mit 15202 Patienten lag die durchschnittliche Gewichtszunahme bei $2,1 \mathrm{~kg}$ im ersten Jahr der Therapie ( $-0,85 \mathrm{~kg}$ bis $+7,5$ $\mathrm{kg})$. Die Zunahme war abhängig vom Insulinregime (basale Therapie weniger als prandiale), der Therapiedauer, dem basalen $\mathrm{HbA}_{1 \mathrm{c}}$ und seiner Veränderung sowie der Hypoglykämiefrequenz. Keinen Einfluss auf die Gewichtszunahme hatte das Ausgangskörpergewicht. spa Miele I et al. Diabetologia 2010;53(Suppl 1) S23. OP 40 